\title{
Non-thermalizability of a Quantum Field Theory
}

\author{
C. R. Hagen* \\ Department of Physics and Astronomy \\ University of Rochester \\ Rochester, N.Y. 14627
}

\begin{abstract}
The problem of understanding the role of large gauge transformations in thermal field theories has recently inspired a number of studies of a one dimensional field theory. Such work has led to the conclusion that gauge invariance is restored only when the entire perturbation expansion can be summed. A careful reexamination of that model is shown, however, to lead to vastly different conclusions when the constraint implied by the field equations is explicitly taken into account. In particular it is found that none of the relevant propagators has any temperature dependence and that the effective action is essentially trivial. A generalization of the model to include bosons as well as fermions is also solved with qualitatively identical results being obtained.
\end{abstract}

A cause of considerable concern in the analysis of Chern-Simons field theories in which a coupling constant quantization mechanism is at work is the fact that induced correction terms are found to be temperature dependent. In other words when such a field theory is thermalized the effective Chern-Simons term is found to have a coefficient which varies smoothly with the temperature, most typically through a term of the form $\tanh (\beta M / 2)$. Since the argument for coupling constant quantization is essentially based upon large gauge transformations, such temperature dependence suggests that the calculational program does not preserve gauge invariance under this class of transformations.

This problem has recently been addressed in a much cited paper [1] which considers a $(0+1)$ dimensional field theory of $N_{f}$ fermions $\psi_{j}, j=1, \ldots, N_{f}$ minimally coupled to a $U(1)$ gauge field $A[2]$. In terms of a real time formulation the Lagrange density is

$$
\mathcal{L}=\sum_{j=1}^{N_{f}} \psi_{j}^{\dagger}\left(i \partial_{t}-m-A\right) \psi_{j}+\kappa A
$$

with $m$ being referred to as a mass parameter. The last term in (1) is a linear ChernSimons term appropriate to a $(0+1)$ dimensional theory. The Lagrangian implies the equation of motion

$$
\left(i \partial_{t}-m-A\right) \psi_{j}=0
$$

and the constraint equation 


$$
\kappa=Q
$$

where[3]

$$
Q=\sum_{j=1}^{N_{f}} \psi_{j}^{\dagger} \psi_{j} .
$$

The only nonvanishing equal time (anti)commutation relation is given by

$$
\left\{\psi_{i}, \psi_{j}^{\dagger}\right\}=\delta_{i j}
$$

with $\psi_{j}$ having the additional property that it annihilates the ground state $\mid 0>$, i.e.,

$$
\psi_{j} \mid 0>=0 .
$$

Although Eq.(3) clearly cannot be valid in the entire Fock space associated with the operators $\psi_{j}$, it can be realized on a reduced physical state space of fixed charge $\kappa$. This leads to the further conclusion that $\kappa$ can assume only positive integer values $n$ as a consequence of the constraint (3) together with (4) and (5). Specifically, for an arbitrary state $\mid>$ Eqs.(3) and (4) imply that the equation

$$
\kappa|>=Q|>
$$

is consistent only for states $\mid>$ which have integer $n$ total charge with $n$ equal to the allowed value of $\kappa[4]$. Thus the $\kappa$ quantization of this work and that of ref. 1 have totally different origins; namely, it follows from the constraint equation here and from topological arguments in the latter.

It was found in ref. 1 that the thermalized version of this theory yields an effective action which is a highly nontrivial function of the temperature, the $m$ parameter, and the integral over $A$. It has the property that its expansion in powers of $A$ has the continuous dependence upon temperature typical of $(2+1)$ dimensional perturbation theory even though the exact version is consistent with gauge invariance. This result has been the basis of a number of studies in $(2+1)$ dimensions [5-8] which depend on specially constructed models which more or less factorize into a $(0+1)$ dimensional part and a two dimensional Euclidean one. Despite the fact that all those results seem to be eminently reasonable, it will be seen here that the $(0+1)$ dimensional model upon which they all ultimately depend is found to have a totally different solution when the constraint (3) is taken into account.

To demonstrate the claimed result it is well to point out at the outset that the model (1) is properly described as a Schrödinger model (or better, a Galilean invariant one) rather than as a $(0+1)$ dimensional theory in the context of special relativity. This conclusion is hard to avoid in view of the fact that there are no antiparticles in the theory and consequently no charge conjugation operator. Once this observation is made one is naturally led to the interpretation of $m$ not as a mass parameter, but 
rather as an internal energy. This means that there is an energy "barrier" $m$ which has to be overcome in the creation of each fermion. Since, however, only states of charge $n$ are allowed by the constraint, the total energy barrier $n m$ amounts merely to a trivial redefinition of the zero point of energy[9]. In terms of operators one can make the change of variable

$$
\psi_{j}^{\prime}(t)=e^{i m t} \psi_{j}(t)
$$

thereby totally eliminating dependence upon the parameter $m$. Since the charge operator is also independent of $m$, one is left with the apparently paradoxical situation that the effective action is required by general considerations to be $m$ independent even though explicit calculations based upon thermal field theory[1,2] lead to the opposite conclusion. It remains to be shown how one can eliminate this seeming contradiction.

One begins the calculation of the effective action with an evaluation of the various fermionic propagators. These are defined in terms of the time ordered products by

$$
S\left(t, t^{\prime}\right)=i \epsilon\left(t, t^{\prime}\right) \frac{\operatorname{Tr} e^{-i H T}\left(\psi_{j}(t) \psi_{j}^{\dagger}\left(t^{\prime}\right)\right)_{+}}{\operatorname{Tr} e^{-i H T}}
$$

where $0 \leq t, t^{\prime} \leq T$ and $\epsilon\left(t, t^{\prime}\right)$ is the alternating function. The trace is to be taken over the space of physical states, namely those of fixed total charge $n$. By letting $T \rightarrow-i \beta$ where $\beta$ is the reciprocal temperature one obtains the usual thermal propagator. Note also that the index $j$ on $S$ has been suppressed since the fields $\psi_{j}$ all have the same $m$ value. Upon using the form

$$
H=m \sum_{j=1}^{N_{f}} \psi_{j}^{\dagger} \psi_{j}
$$

for the Hamiltonian and the fact that for all allowed states of the system $H$ has the constant value $\mathrm{nm}$ the expression for the propagator reduces to the $T$ independent form

$$
S\left(t, t^{\prime}\right)=i \epsilon\left(t, t^{\prime}\right) \frac{\operatorname{Tr}\left(\psi_{j}(t) \psi_{j}^{\dagger}\left(t^{\prime}\right)\right)_{+}}{\operatorname{Tr} 1} .
$$

Physically, this $T$ independence arises, of course, from the fact that the thermal averaging is carried out only over a set of states all of which are degenerate in energy with each other. Thus the constraint leads directly to the claimed result that this theory cannot be thermalized in the usual fashion. It may be noted that if one allows the various particles in the theory to have different $m$ (i.e., internal energy) values, a $T$ dependence can be forced into the model. However, the effective action will not display such $T$ dependence and will in fact coincide with the degenerate $m$ case.

It is clearly of interest to explain at this point why the usual thermalized propagators are not valid in the present case. To this end it should be noted that the 
standard derivation depends upon two assumptions, neither of which is valid for this model. The first of these is that the Hamiltonian generates the time dependence of the fermion operators; namely, that

$$
\psi_{j}(t)=e^{i H t} \psi_{j}(0) e^{-i H t} .
$$

However, this equation is certainly incorrect in the application at hand since it fails to reproduce the correct equation of motion. More specifically, one notes that $\psi_{j}$ depends on the integral of $A(t)$ and thus the commutator of $H$ with $\psi_{j}$ fails to give its time derivative. A second reason for the failure of the usual derivation of the thermal propagator is that it requires the validity of the expression

$$
\operatorname{Tr} A B=\operatorname{Tr} B A
$$

which is not tenable for this model. The reason is that although bilinear operators such as the charge are well defined, one violates (6) in giving meaning to the operator $\psi_{j}$ which has the property of taking one out of the space of physical states. Thus one finds that any attempt to derive the usual thermal boundary conditions by application of Eq.(6) in effect transforms the trace over the physical state space into one over an unphysical set of states.

To calculate the propagator one begins with the $A=0$ case which will be designated by $S_{0}\left(t, t^{\prime}\right)$. It can be written as

$$
S_{0}\left(t, t^{\prime}\right)=i \theta_{+}\left(t, t^{\prime}\right)<0\left|\psi_{j}(t) \psi_{j}^{\dagger}\left(t^{\prime}\right)\right| 0>-i \theta_{-}\left(t, t^{\prime}\right)<0\left|\psi_{j}^{\dagger}\left(t^{\prime}\right) \psi_{j}(t)\right| 0>
$$

where $\theta_{ \pm}(x)=\frac{1}{2}\left(1 \pm \frac{x}{|x|}\right)$. This readily leads to the result

$$
S_{0}\left(t, t^{\prime}\right)=i\left[\theta_{+}\left(t, t^{\prime}\right)-\frac{n}{N_{f}}\right] e^{-i m\left(t-t^{\prime}\right)} .
$$

It may be noted that this differs from ref. 2 only in that in the latter case $n$ is a function of temperature while here it is simply a nonnegative integer.

The effect of the interaction can now be included by considering the equation for the $A \neq 0$ propagator

$$
\left(-i \partial_{t}+m\right) S\left(t, t^{\prime}\right)=\delta\left(t-t^{\prime}\right)-A(t) S\left(t, t^{\prime}\right)
$$

Although it might be supposed that this equation is to be solved perturbatively by expansion of the formal expression

$$
S=S_{0}\left(1+A S_{0}\right)^{-1}
$$

with $S_{0}$ given by Eq.(7), this is yet another instance in which the usual assumptions fail. The essential point here is that correct boundary conditions on the exact propagator cannot be ensured by the imposition of an appropriate set of boundary 
conditions on the inverse of the operator $-i \partial_{t}+m$, thereby leading to an acceptable solution of the form (8). This stands in marked contrast to the case of relativistic field theory in which the invocation of causal boundary conditions (i.e., positive frequencies in the future, negative frequencies in the past) on the non-interacting propagators automatically ensures that such conditions will characterize the entire perturbation series. Similarly, the imposition of causal (i.e., retarded) or anti-causal (advanced) boundary conditions in a Galilean theory will suffice to guarantee that they will be satisfied by the exact solution.

One obtains a solution for the exact propagator in either of two different ways. The more basic approach merely recognizes that the equation for this function is simply a first order differential equation in a single variable which must therefore be soluble by elementary means. The result is

$$
S\left(t, t^{\prime}\right)=S_{0}\left(t, t^{\prime}\right) \exp \left[-i \int_{t^{\prime}}^{t} A(\tau) d \tau\right]
$$

Alternatively, one could derive (9) by an approach which is close in spirit to the result (8). However, it is first necessary to split $S\left(t, t^{\prime}\right)$ into two parts so that proper boundary conditions can be imposed. Thus one defines

$$
S_{ \pm}\left(t, t^{\prime}\right)=\theta_{ \pm}\left(t, t^{\prime}\right) S\left(t, t^{\prime}\right)
$$

and notes that these functions satisfy the equations

$$
\left(-i \partial_{t}+m+A\right) S_{+}\left(t, t^{\prime}\right)=\left(1-\frac{n}{N_{f}}\right) \delta\left(t-t^{\prime}\right)
$$

and

$$
\left(-i \partial_{t}+m+A\right) S_{-}\left(t, t^{\prime}\right)=\frac{n}{N_{f}} \delta\left(t-t^{\prime}\right)
$$

Since $S_{+}\left(t, t^{\prime}\right)$ and $S_{-}\left(t, t^{\prime}\right)$ clearly must satisfy retarded and advanced boundary conditions respectively, it is now straightforward to obtain solutions of these equations as

$$
S_{+}=\left(1-\frac{n}{N_{f}}\right) S_{0+}\left(1+A S_{0+}\right)^{-1}
$$

and

$$
S_{-}=\frac{n}{N_{f}} S_{0-}\left(1+A S_{0-}\right)^{-1}
$$

where

$$
S_{0 \pm}\left(t, t^{\prime}\right)= \pm i \theta_{ \pm}\left(t, t^{\prime}\right) e^{-i m\left(t-t^{\prime}\right)} .
$$

Upon combining the solutions $S_{ \pm}$one obtains the result 


$$
S\left(t, t^{\prime}\right)=S_{+}\left(t, t^{\prime}\right)+S_{-}\left(t, t^{\prime}\right) .
$$

From Eq.(10) it follows that

$$
\begin{aligned}
S_{+}\left(t, t^{\prime}\right)= & \left(1-\frac{n}{N_{f}}\right) S_{0+}\left(t, t^{\prime}\right) \sum_{r=0}^{\infty}(-i)^{r} \int d \tau_{1} \int d \tau_{2} \ldots \int d \tau_{r} A\left(\tau_{1}\right) A\left(\tau_{2}\right) \ldots A\left(\tau_{r}\right) \\
& \theta_{+}\left(t-\tau_{1}\right) \theta_{+}\left(\tau_{1}-\tau_{2}\right) \ldots \theta_{+}\left(\tau_{r}-t^{\prime}\right)
\end{aligned}
$$

However, this is clearly equivalent to

$$
S_{+}\left(t, t^{\prime}\right)=\left(1-\frac{n}{N_{f}}\right) S_{0+}\left(t, t^{\prime}\right) \exp \left[-i \int_{t^{\prime}}^{t} A(\tau) d \tau\right] .
$$

Corresponding manipulation of Eq.(11) yields

$$
S_{-}\left(t, t^{\prime}\right)=\frac{n}{N_{f}} S_{0-}\left(t, t^{\prime}\right) \exp \left[-i \int_{t^{\prime}}^{t} A(\tau) d \tau\right] .
$$

Upon combining these results for $S_{ \pm}\left(t, t^{\prime}\right)$ one obtains the result (9), thereby demonstrating the equivalence of the two approaches.

It is now straightforward to obtain the effective action as a function of $A$. To this end one notes that

$$
<Q>i N_{f} \lim _{\epsilon \rightarrow 0} S\left(t, t^{\prime}=t+\epsilon\right)
$$

which from (7) and (9) is readily seen to yield

$$
<Q>=n
$$

in agreement with the constraint (3). Thus the effective action $\Gamma(A)$ has the form

$$
\Gamma(A)=\operatorname{in} \int A(\tau) d \tau
$$

a result which stands in marked contrast to what has been obtained in treatments which do not take into account the constraint (3).

The model considered here can be extended to include bosons as well as fermions[10]. Such a system is obtained by the replacement of (1) by

$$
\mathcal{L}=\sum_{j=1}^{N_{f}} \psi_{j}^{\dagger}\left(i \partial_{t}-m-A\right) \psi_{j}+\sum_{j=1}^{N_{b}} \phi_{j}^{\dagger}\left(i \partial_{t}-\mu-A\right) \phi_{j}+\kappa A .
$$

The boson operators $\phi_{j}, j=1, \ldots, N_{b}$ have the nonvanishing equal time commutators

$$
\left[\phi_{i}, \phi_{j}^{\dagger}\right]=\delta_{i j}
$$


and the property that

$$
\phi_{j} \mid 0>=0 \text {. }
$$

The total charge operator $Q$ now has the form

$$
Q=\sum_{j=1}^{N_{f}} \psi_{j}^{\dagger} \psi_{j}+\sum_{j=1}^{N_{b}} \phi_{j}^{\dagger} \phi_{j}
$$

with the constraint (3) being unmodified when expressed in terms of the charge (13). As before one concludes that $\kappa$ must be a nonnegative integer $n$, although unlike the purely fermionic case it is no longer bounded from above.

By assuming that the fermionic and bosonic internal energies $m$ and $\mu$ are unequal one can obtain propagators which have a temperature dependence[11]. Despite such dependence the arguments against using the usual thermal propagators remain valid and one obtains the correct result by combinatorial arguments. Thus for the $A=0$ fermionic propagator $S_{0}\left(t, t^{\prime}\right)$ one finds that the Hamiltonian

$$
H=m \sum_{j=1}^{N_{f}} \psi_{j}^{\dagger} \psi_{j}+\mu \sum_{j=1}^{N_{b}} \phi_{j}^{\dagger} \phi_{j}
$$

implies the result

$$
S_{0}\left(t, t^{\prime}\right)=i\left[\theta_{+}\left(t, t^{\prime}\right)-\frac{i}{N_{f}} \frac{\partial}{\partial(m T)} \log C\right] e^{-i m\left(t-t^{\prime}\right)}
$$

where

$$
C=\sum_{r=0}^{N} e^{-i[r m+(n-r) \mu] T} \frac{N_{f} !}{r !\left(N_{f}-r\right) !} \frac{\left(N_{b}+n-r-1\right) !}{\left(N_{b}-1\right) !(n-r) !}
$$

where $N$ is the lesser of $n$ and $N_{f}$. The $\phi$ propagators $D\left(t, t^{\prime}\right)$ are defined as

$$
D\left(t, t^{\prime}\right)=i \frac{\operatorname{Tr} e^{-i H T}\left(\phi_{j}(t) \phi_{j}^{\dagger}\left(t^{\prime}\right)\right)_{+}}{\operatorname{Tr} e^{-i H T}}
$$

and are found in the $A=0$ limit to be

$$
D_{0}\left(t, t^{\prime}\right)=i\left[\theta\left(t, t^{\prime}\right)+\frac{1}{N_{b}}\left(n-i \frac{\partial}{\partial(m T)} \log C\right)\right] e^{-i \mu\left(t-t^{\prime}\right)} .
$$

As before one finds that

$$
S\left(t, t^{\prime}\right)=S_{0}\left(t, t^{\prime}\right) \exp \left[-i \int_{t^{\prime}}^{t} A(\tau) d \tau\right]
$$

and

$$
D\left(t, t^{\prime}\right)=D_{0}\left(t, t^{\prime}\right) \exp \left[-i \int_{t^{\prime}}^{t} A(\tau) d \tau\right] .
$$


This implies that the matrix element of the charge operator as given by

$$
<Q>=i N_{f} \lim _{\epsilon \rightarrow 0} S\left(t, t^{\prime}=t+\epsilon\right)-i N_{b} \lim _{\epsilon \rightarrow 0} D\left(t, t^{\prime}=t+\epsilon\right)
$$

reduces to

$$
<Q>=n
$$

so that the effective action is the same as in the purely fermionic case. Thus one finds that the inclusion of bosonic fields, while providing a natural mechanism for the introduction of temperature dependence into the various propagators, has the property of leaving the matrix elements of the charge operator (and hence the effective action) unchanged and temperature independent just as in the original model. One is forced to conclude that the laudable goal of finding a mechanism to explain the disturbing temperature dependence in $(2+1)$ dimensional Chern-Simons theories is not to be realized in the thermalization of the model (1).

The author acknowledges useful discussions with Dr. O. Kong. This work is supported in part by the U.S. Department of Energy Grant No.DE-FG02-91ER40685.

*Electronic address: hagen@urhep.pas.rochester.edu 


\section{References}

1. G. Dunne, K. Lee, and C. Lu, Phys. Rev. Lett. 78, 3434 (1997).

2. Additional study of this model is to be found in A. Das and G. Dunne, hepth/9712144.

3. It should be noted that the definition of $Q$ in ref. 1 differs from that used here by the term $N_{f} / 2$ so that the charge of the state $\mid 0>$ has the more conventional value of zero in the present work.

4. Because of the fermionic statistics $n$ must satisfy the condition $0 \leq n \leq N_{f}$.

5. S. Deser, L. Griguolo, and D. Seminara, Phys. Rev. Lett. 79, 1976 (1997).

6. C. D. Fosco, G. L. Rossini, and F. A. Schaposnik, Phys. Rev. Lett. 78, 1980 (1997).

7. I. J. R. Aitchison and C. Fosco, hep-th/9709035.

8. R. González-Felipe, hep-th/9709079.

9. In the event that particles of different masses can transmute into each other, the differences between the zero points of internal energy for various particles can have observable significance. An example of this occurs in the Galilean Lee model which has been considered by J. M. Lévy-Leblond, Commun. math Phys. 6, 286 (1967).

10. The study of such a system was in fact suggested in ref.1.

11. Just as in the fermionic case all the internal energies can be transformed away. This will not be done here in order to emphasize the fact that the effective action is temperature independent regardless of whether one carries out such a step. 